\title{
A New Paradigm on the TeV-scale Cosmic Rays: Contributions from the local sources
}

\author{
Yong-Yeon Keum* \\ Author affiliation: Center for Theoretical Physics, Department of Physics, Seoul National \\ University, 1 Kwan-Ak Ro, Kwan-Ak Gu, Seoul, Republic of Korea \\ E-mail: yykeum2011esnu.ac.kr

\section{Jihyeun Bang} \\ Affiliation: Department of Physics, Seoul National University, 1 Kwan-Ak Ro, Kwan-Ak Gu, \\ Seoul, Republic of Korea \\ E-mail: coolbang115@snu.ac.kr
}

\begin{abstract}
Recent measurements of cosmic ray proton and helium spectra in CREAM, PAMELA and AMS02 experiments show a hardening above a few hundreds of GeV. This excess is hard to understand in the framework of the conventional models of Galactic cosmic ray production and propagation. We propose here to explain this anomaly by the presence of local sources. Cosmic ray propagation is described as a diffusion process taking place inside a two-zone magnetic halo. We calculate the proton and helium fluxes at the Earth between $50 \mathrm{GeV}$ and $100 \mathrm{TeV}$. Improving over a similar analysis, we consistently derive these fluxes by taking into account both local and remote sources for which a unique injection rate is assumed. We find cosmic ray propagation parameters for which the proton and helium spectra remarkably agree with the CREAM, PAMELA and AMS02 measurements over four decades in energy.
\end{abstract}

The 39th International Conference on High Energy Physics (ICHEP2018)

4-11 July, 2018

Seoul, Korea

${ }^{*}$ Speaker. 


\section{Observations of the CR proton and helium anomaly.}

Recent measurements of the absolute high-energy CR proton and helium spectra have been reported by CREAM [1,2] and PAMELA [3] experiments. Observations point towards an excess in the $\mathrm{CR}$ proton and helium fluxes above $250 \mathrm{GeV} /$ nuc. The single power-law hypothesis is rejected at $95 \%$ C.L. The hardening of the proton spectrum occurs at $232_{-30}^{+35} \mathrm{GeV}$ with a change of the spectral index from $2.85 \pm 0.015 \pm 0.004$ to $2.67 \pm 0.03 \pm 0.05$. For the helium data, the spectral index varies from $2.766 \pm 0.01 \pm 0.027$ to $2.477 \pm 0.06 \pm 0.03$ with the hardening setting in at $243_{-31}^{+27} \mathrm{GeV} /$ nuc. These results challenge the conventional scenarios proposed so far to model Galactic cosmic rays. They indicate the presence of an anomalous behavior of the CR proton and helium spectra in the $100 \mathrm{GeV}$ to $100 \mathrm{TeV}$ energy range.

\section{Our explanation of the proton and helium anomaly.}

In this talk, we show that the proton and helium spectral hardening above $250 \mathrm{GeV} /$ nuc can be attributed to local sources of cosmic rays, whose presence is associated to known supernova remnants (SNR) and pulsars. These objects can be found in astronomical catalogs such as the Green catalog [4] which can be completed with the ATNF pulsar database[5, 6]. In this case, there is no need to modify the conventional CR propagation model. The principal weakness of these analyses is the lack of a consistent treatment of the CR spectra in the entire energy range extending from tens of $\mathrm{GeV}$ up to a few PeV. It should be noted that the magnitude of the CR proton (helium) flux is related over the entire energy range to the injection rate $q$ of individual sources. The low energy (power-law regime) and high energy (spectral hardening) parts of the CR spectra are connected with each other. A consistent treatment of the problem requires that the proton and helium fluxes are calculated over the entire energy range. A crucial problem is also to understand why just a few local sources could explain the spectral hardening at high energies whereas the bulk of the Galactic sources is required in order to account for the power-law behavior of the fluxes below $250 \mathrm{GeV} / \mathrm{nuc}$. This aspect, which is not addressed in the above mentioned analyses, bears upon the more general question of the discreet nature of the sources. In the conventional model of CR propagation, these are treated as a jelly spreading over the Galactic disk and continuously accelerating cosmic rays. The question arises then to understand why and in which conditions that scheme breaks down at high energies where local and point-like objects come into play. The results presented here are based on a detailed investigation of that question. Bernard et al. [7, 8] have recently shown how to reconcile the presence of point-like sources with the conventional description of CR production and propagation. Once accelerated by the sources that lie within the Galactic disk, CR nuclei diffuse on the irregularities of the Galactic magnetic field. The diffusion coefficient $K=K_{0} \beta \mathscr{R}^{\delta}$ accounts for that process, where $K_{0}$ is a normalization constant and $\beta$ denotes the particle velocity. The magnetic halo, inside which cosmic rays propagate before escaping into intergalactic space, is assumed to be a flat cylindrical domain which matches the circular structure of the Milky Way. The Galactic disk is sandwiched between two confinement layers whose thickness $L$ is unknown and turns out to be crucial in our investigation. Stellar winds combine to generate a Galactic convection that wipes cosmic rays away from the disk, with velocity $V_{c}(z)=V_{c} \operatorname{sign}(z)$. CR nuclei also undergo collisions with the interstellar medium (ISM) with a rate $\Gamma_{\mathrm{sp}}=\sigma_{\mathrm{col}} \beta n_{\mathrm{ISM}}$. Above a few GeV, diffusive re- 
acceleration and energy losses may be disregarded and the master equation for the space and energy number density $\psi \equiv d n / d T$ of a given CR species simplifies into the diffusion equation

$$
\frac{\partial \psi}{\partial t}+\partial_{z}\left(V_{c} \psi\right)-K(E) \triangle \psi+\Gamma_{\mathrm{sp}} \psi=q_{\mathrm{acc}} .
$$

The CR transport parameters $K_{0}, \delta, L$ and $V_{c}$ can be weakly constrained from the B/C ratio. In the conventional approach, the CR source term $q_{\text {acc }}$ is a continuous function of space and time. Steady-state is moreover assumed. This is an oversimplification insofar as CR sources are actually point-like, with an average supernova explosion rate $v$ of 1 to 3 events per century. In our model, the production rate of $\mathrm{CR}$ nuclei through acceleration is given by

$$
q_{\mathrm{acc}}\left(\mathbf{x}_{S}, t_{S}\right)=\sum_{i \in \mathscr{P}} q_{i} \delta^{3}\left(\mathbf{x}_{S}-\mathbf{x}_{i}\right) \delta\left(t_{S}-t_{i}\right)
$$

where each source $i$ that belongs to the population $\mathscr{P}$ contributes a factor $q_{i}$ at position $\mathbf{x}_{i}$ and time $t_{i}$. The total flux $\Phi \equiv(1 / 4 \pi) \beta \psi$ at the Earth depends on the precise locations and ages of all the sources and varies from one particular population $\mathscr{P}$ to another. Because we do not know the actual distribution of the Galactic sources that have generated the observed CR flux, we must rely on a statistical analysis and consider the position and age of each source as random variables. The CR flux $\Phi(E)$ at a given energy $E$ behaves as a stochastic variable whose probability distribution function $p(\Phi)$ has been studied in [7,8]. The conventional CR model is recovered by taking the statistical average of the flux over the ensemble of all possible populations $\mathscr{P}$. This average flux $\bar{\Phi}$ turns out to be the solution of Eq. 2.1 with a continuous source term $q_{\text {acc }}$. More exciting is the spread of the flux $\Phi$ around its average value $\bar{\Phi}$. According to this line of reasoning, the proton and helium anomaly results from the particular configuration of the actual CR sources. These objects are incidentally known in the nearby region for which catalogs of SNR and pulsars are available. The domain extending $2 \mathrm{kpc}$ around the Earth and encompassing objects that have exploded less than 30,000 years ago is defined as the local region. The catalogs are no longer complete outside and fail to be reliable. In the conventional CR model, the local sources would yield an average contribution $\bar{\Phi}_{\text {loc }}$ whereas the actual objects yield a much larger flux $\Phi_{\text {cat }}$. Denoting by $\Phi_{\text {ext }}$ the flux from the other sources, we infer a total signal at the Earth

$$
\Phi=\Phi_{\text {cat }}+\Phi_{\text {ext }}
$$

to be compared to the prediction of the conventional steady-state model

$$
\bar{\Phi}=\bar{\Phi}_{\text {loc }}+\bar{\Phi}_{\text {ext }} .
$$

The flux produced by the external sources has a very small variance as shown in Ref. [7]. We may then identify $\Phi_{\text {ext }}$ with its statistical average $\bar{\Phi}_{\text {ext }}$. We have performed a scan over the CR parameters in order to fit the CREAM [1] and PAMELA [3] data over an energy range extending from $50 \mathrm{GeV} /$ nuc to $100 \mathrm{TeV} /$ nuc. For each model, CR propagation is described by $K_{0}, \delta$ and $L$. At high energy, Galactic convection and solar modulation have little effect on the CR flux. That is why we have set $V_{c}$ and the Fisk potential $\phi_{\mathrm{F}}$ equal to 0 during the scan. The injection indices $\alpha_{\mathrm{p}}$ and $\alpha_{\mathrm{He}}$ are independently adjusted to the proton and helium spectra. Observations point towards 
slightly different power laws for these fluxes at energies below $250 \mathrm{GeV} / n u c$. The last injection parameter which we have considered is the average supernova explosion rate $v$ in the Galaxy. Each $\mathrm{CR}$ configuration is then characterized by six parameters. The quality of the fits to the proton and helium data is respectively gauged by the reduced chi-squares $\chi_{\mathrm{p}}^{2}$ and $\chi_{\mathrm{He}}^{2}$. We have found many models which reproduce fairly well the power-law regime and the hardening of both the proton and helium fluxes, as shown in Figures of the previous our works [8, 9] and ICHEP2018 Proceedings [10].

\section{Discussion and conclusion.}

This excellent agreement makes us confident that the proton and helium anomaly can actually be explained by existing local sources which have been extracted from SNR and pulsar surveys. The model which we have presented here is quite simple. Refining it is beyond the scope of this article.

The simplistic solution to the proton and helium anomaly which we have sketched in this article is definitely exciting in spite of the above mentioned problems and should motivate further investigations.

The Green Function Method will be used widely in the analysis of the positron excess by using of the pulsar sources, and the background study of anti-proton flux in indirect search of Dark Matter by using the cosmic ray fluxes.

\section{Acknowledgments}

YYK's previous works have been supported by the Basic Science Research Program through the National Research Foundation of Korea (NRF) funded by the Korean Ministry of Education, Science and Technology (Grant Nos:2013K2A1A2054309; 2013R1A1A2009799), and JB and YYK's recent work is supporting by RNF-2017R1D1A1B03035250.

\section{References}

[1] H.S. Ahn et al. (CREAM collaboration), The Astrophysical Journal 714 L89-L93 (2010).

[2] Y.S. Yoon et al. (CREAM collaboration), The Astrophysical Journal 728122 (2011).

[3] O. Adriani et al. (PAMELA collaboration), Science 33269 (2011).

[4] D.A. Green, (2009), [arXiv:0905.3699], URL http://www.mrao.cam.ac.uk/surveys/snrs.

[5] R.N. Machester, C.B. Hobbs, A. Teoh, and M. Hobbs, The Astronomical Journal 129, 1993 (2005).

[6] ATNF pulsar catalogue, http://www.atnf.csiro.au/research/pulsar/psrcat/.

[7] G. Bernard, T. Delahaye, p. Salati and R. Taillet, Astronomy \& Astrophysics, 544, A92 (2012).

[8] G. Bernard, T. Delahaye, Y.-Y. Keum, W. Liu, P. Salati, and R. Taillet, Astronomy \& Astrophysics 555, A48 (2013).

[9] Yong-Yeon Keum and P. Salati, PARAMANA-journal of physics, Vol 86, No 2, pp369-377 (2016).

[10] Jihyeun Bang and Yong-Yeon Keum, PoS Proceedings of ICHEP2018, 785(2018). 